\title{
Palonosetron in combination with 1-day versus 3-day dexamethasone for prevention of nausea and vomiting following moderately emetogenic chemotherapy: a randomized, multicenter, phase III trial
}

\author{
Luigi Celio • Sergio Frustaci • Angela Denaro • \\ Angela Buonadonna $\cdot$ Antonio Ardizzoia • \\ Elena Piazza • Alessandra Fabi • \\ Alba Maria Capobianco $\cdot$ Luciano Isa • \\ Luigi Cavanna • Alessandro Bertolini • Ettore Bichisao • \\ Emilio Bajetta • \\ for the Italian Trials in Medical Oncology Group
}

Received: 10 February 2010 / Accepted: 14 June 2010 /Published online: 25 June 2010

(C) The Author(s) 2010. This article is published with open access at Springerlink.com

\begin{abstract}
Purpose A phase III trial assessed the efficacy of palonosetron plus dexamethasone given once in preventing acute and delayed chemotherapy-induced nausea and vomiting (CINV) following a broad range of moderately emetogenic chemotherapy (MEC) regimens.

Methods This multicentre, randomized, open-label, noninferiority trial evaluated two different treatment groups.
\end{abstract}

L. Celio $(\bowtie) \cdot$ A. Denaro $\cdot$ E. Bajetta

Medical Oncology Unit 2,

Fondazione IRCCS "Istituto Nazionale dei Tumori”,

Via Venezian 1,

20133 Milan, Italy

e-mail: luigi.celio@istitutotumori.mi.it

S. Frustaci $\cdot$ A. Buonadonna

Centro di Riferimento Oncologico,

Via Gallini 2,

33081 Aviano, Italy
A. Ardizzoia
Ospedale San Gerardo,
Via Pergolesi 33,
20052 Monza, Italy
E. Piazza
Ospedale L. Sacco,
Via Grassi 74,
20157 Milan, Italy

\author{
A. Fabi \\ Istituto Regina Elena, \\ Via Chianesi 53, \\ 00144 Rome, Italy
}

One group received palonosetron $(0.25 \mathrm{mg}$ intravenously) and dexamethasone ( $8 \mathrm{mg}$ intravenously) before chemotherapy, while the other was administered the same regimen on day 1 followed by dexamethasone $8 \mathrm{mg}$ orally on days 2 and 3 . The primary endpoint was complete response (CR; defined as no emetic episodes and no rescue medication) during the overall phase (days 1-5 after chemotherapy initiation). The non-inferiority margin

A. M. Capobianco

Centro Riferimento Oncologico della Basilicata,

Strada Provinciale n. 8 del Vulture,

85028 Rionero in Vulture, Italy

L. Isa

Ospedale Serbelloni,

Via Bellini 5,

20064 Gorgonzola, Italy

L. Cavanna

Ospedale Guglielmo da Saliceto,

Via Taverna 49,

29100 Piacenza, Italy
A. Bertolini
Ospedale di Sondrio,
Via Stelvio 25,
23100 Sondrio, Italy
E. Bichisao
ITMO operative office,
Via Ponzio 44,
20133 Milan, Italy 
was predefined as a $15 \%$ difference between groups in the primary endpoint.

Results Of 332 chemotherapy-naïve patients included in the intention-to-treat analysis, $65.1 \%$ were female, and $35.2 \%$ received anthracycline plus cyclophosphamide (AC)-based regimens. Overall CR rates were $67.5 \%$ for those administered dexamethasone only on day $1(n=166)$, and $71.1 \%$ for those also administered dexamethasone on days 2 and 3 ( $n=166$; difference $-3.6 \%$ (95\% confidence interval, -13.5 to 6.3)). CR rates were not significantly different between groups during the acute ( $0-24 \mathrm{~h}$ post-chemotherapy; $88.6 \%$ versus $84.3 \% ; P=0.262$ ) and delayed phases (days $2-5$; $68.7 \%$ versus $77.7 \% ; P=0.116$ ).

Conclusions Palonosetron plus single-dose dexamethasone administered before common MEC regimens provide protection against acute and delayed CINV which is noninferior to that of palonosetron plus dexamethasone for 3 days. However, the major benefit of the single-day regimen occurs in patients receiving non-AC MEC regimens.

Keywords Palonosetron - Serotonin antagonists . Dexamethasone $\cdot$ Moderately emetogenic chemotherapy . Nausea $\cdot$ Vomiting

\section{Introduction}

Chemotherapy-induced nausea and vomiting (CINV) can severely impact performance status and quality of life [1]. CINV has been traditionally categorized as acute or delayed: acute CINV is that occurring within $24 \mathrm{~h}$ after initiation of chemotherapy, whereas delayed emesis is that occurring between days 2 and 5 [2]. The risk of CINV depends on several factors, although the emetogenic potential of the chemotherapeutic agent is the most predictive factor [3]. Intravenously administered cytotoxic agents were initially assigned to five levels of emetogenicity that was subsequently divided into four emetic risk categories (high, moderate, low, and minimal) $[4,5]$. A critical factor in guiding anti-emetic treatment is the ability of chemotherapy to induce a substantial risk of delayed emesis. There is limited knowledge of the potential for delayed emesis of many moderately emetogenic agents, but recent guidelines support the use of a serotonin $\left(5-\mathrm{HT}_{3}\right)$ receptor antagonist plus dexamethasone before moderately emetogenic chemotherapy (MEC), with either agent given alone on days 2 and 3 [6]. A meta-analysis of results from 32 trials that included 5,613 patients who received highly emetogenic chemotherapy or MEC indicated that dexamethasone is superior to placebo or no treatment for complete protection against both acute and delayed CINV [7]. Most clinical trials also found that the addition of dexamethasone is more effective than a 5- $\mathrm{HT}_{3}$-receptor antagonist alone for control of acute and delayed emesis [2]. However, dexamethasone for prophylaxis of delayed emesis after MEC induces moderate-to-severe adverse effects that may have substantial impact on the quality of life [8]. In addition, the firstgeneration $5-\mathrm{HT}_{3}$-receptor antagonists have only modest efficacy in prevention of delayed emesis induced by MEC [9].

Palonosetron is a second-generation $5-\mathrm{HT}_{3}$-receptor antagonist that has a greater binding affinity and a prolonged half-life $(\sim 40 \mathrm{~h})$ compared with first-generation agents [10]. In two randomized, double-blind, controlled, phase III, non-inferiority trials involving patients receiving MEC, single-dose palonosetron ( $0.25 \mathrm{mg}$ intravenously) produced a statistically significant increase in the complete response (CR) rate compared with single-dose ondansetron or dolasetron for CINV during the delayed and overall phases [11, 12]. Pooled analysis of these trials showed that palonosetron also induces a statistically significant improvement in the CR rate during the acute phase compared with pooled data from patients who received ondansetron or dolasetron $(72 \%$ versus $61 \%$, respectively) [13]. Significantly more patients receiving palonosetron achieved complete protection from delayed CINV compared with those who received either ondansetron or dolasetron ( $64 \%$ versus $47 \%$, respectively). It is well known that the addition of dexamethasone to a $5-\mathrm{HT}_{3}$ receptor antagonist improves the anti-emetic efficacy during the acute phase, control of which is the strongest prognostic factor for successful prevention of delayed CINV $[2,14]$. In a recent open-label, phase II study evaluating the combination of palonosetron and dexamethasone $(8 \mathrm{mg})$ administered intravenously on day 1 in patients receiving MEC, CR was seen in $84 \%$ of patients during the acute phase [15].

Based on this data, palonosetron plus a single dose of dexamethasone administered before chemotherapy might provide a more convenient regimen for the prevention of acute and delayed CINV following MEC regimens. Accordingly, the present phase III trial was designed to test the hypothesis that the efficacy of a single-day twodrug regimen against $\mathrm{CINV}$ is non-inferior to that of the same regimen with additional dexamethasone doses on days 2 and 3 after chemotherapy initiation.

\section{Patients and methods}

Study design

This was a phase III, randomized, open-label, parallelgroup, active-comparator, non-inferiority trial (EudraCT number 2006-000644-13). Patients were enrolled in 15 centers in Italy coordinated by the Italian Trials in Medical Oncology (I.T.M.O.) Group. This was an unsponsored, investigator-initiated trial. Design and methodology of this trial, with the exception of blinding, were similar to those 
of the two phase III registration studies that assessed the efficacy of palonosetron alone in patients receiving MEC $[11,12]$. The institutional review board at each study site approved the study protocol, and patients provided written informed consent prior to participation.

\section{Eligibility criteria}

Patients eligible for the study were adults with a histologically or cytologically confirmed solid tumor receiving chemotherapy for the first time with intravenous agents classified as moderately emetogenic according to the modified Hesketh chemotherapy classification given as single doses on study day 1 [5]. Patients were required to have acceptable hematologic, hepatic, and renal functions for administration of chemotherapy, and an adequate Eastern Cooperative Oncology Group (ECOG) performance status of 0,1 , or 2 . Patients could not receive any drug with potential anti-emetic efficacy other than the study drug given immediately before or during the study period, and could not receive radiotherapy within 30 days before chemotherapy initiation or during the study period. Patients were considered ineligible if they had known central nervous system malignancy or another organic cause for nausea or vomiting unrelated to chemotherapy administration. Nausea or vomiting within $24 \mathrm{~h}$ prior to initiation of chemotherapy also led to exclusion. Patients were ineligible if they had active infection or were unable to understand or cooperate with study procedures. Pregnant or nursing women were ineligible.

Treatment plan and random assignment

Regardless of assignment to either study arm, all patients received a single, fixed, intravenous dose of palonosetron $(0.25 \mathrm{mg})$ as a bolus over $30 \mathrm{~s}$ given $30 \mathrm{~min}$ before chemotherapy initiation on day 1 . Administration of prophylactic dexamethasone (8 $\mathrm{mg}$ intravenously) within $15 \mathrm{~min}$ before chemotherapy initiation on day 1 was also required. Patients were randomly assigned to one of two delayed anti-emetic regimens: (1) no additional dexamethasone, or (2) dexamethasone ( $8 \mathrm{mg}$ orally) on days 2 and 3. After chemotherapy, rescue medication including dexamethasone and/or metoclopramide for the treatment of nausea and vomiting was permitted on an as-needed basis. Patients who met eligibility criteria were randomly assigned to either treatment arm using an allocation schedule with a block-size of four that had been generated before the trial by an independent statistician.

Study endpoints and assessments

The primary efficacy endpoint of this study was CR (defined as no emetic episodes, and no rescue medication use) during the overall study period (days 1 through 5 after chemotherapy initiation). Secondary endpoints were the proportion of patients who achieved the following during the overall, acute ( $0-24 \mathrm{~h}$ post-chemotherapy), and delayed phase (24-120 h post-chemotherapy): CR (not including overall phase), complete control (CC; defined as no emetic episodes, no use of rescue medication, and no more than mild nausea), no emesis (defined as no vomiting or retching), no nausea, and no use of rescue medication. An additional endpoint was severity of nausea that was recorded on a four-point categorical Likert scale $(0$, none; 1, mild; 2 , moderate; 3 , severe), according to subjective assessment by each patient.

Patients were instructed to record the relevant study information in a diary provided by the investigators. To assess efficacy, patients made daily entries in the diary for 5 days after chemotherapy initiation to record the number and time of any emetic events in the previous $24 \mathrm{~h}$, use of rescue medication, and maximum nausea experienced in the previous $24 \mathrm{~h}$ assessed by a Likert scale. Patients made daily entries in the diary noting any adverse event or use of concomitant medication. When patient returned to the clinic for the second course of chemotherapy, the patient diary was collected and reviewed to ensure adherence to the required diary documentation and the prescribed study medication at home. Adverse events judged by the investigator to be possibly, probably, or definitely related to the study treatment were regarded as a treatment-related adverse event.

\section{Statistical analysis}

The intention-to-treat (ITT) cohort included patients who received at least one dose of the study medication and MEC chemotherapy. The per-protocol (PP) cohort included all patients who received study medication and completed the follow-up period (days 1-5 after chemotherapy initiation) without any major protocol deviation. The safety cohort included all patients who received at least one dose of the study medication after randomization. The primary efficacy hypothesis was that palonosetron plus a single-dose of dexamethasone was non-inferior to palonosetron plus dexamethasone for 3 days during the overall study period $(0-120 \mathrm{~h}$ post-chemotherapy) using a non-inferiority margin of a $15 \%$ difference between groups in the proportion of patients who had a CR. Considering previous pivotal studies, the number of patients needed in the study was estimated to be 330 , who were distributed into two groups (i.e., 165 patient/group) based on the assumption of a CR rate of $70 \%$ in the two treatment groups and a difference of $-15 \%$ in the $\mathrm{CR}$ rate $[11,12]$. To obtain $80 \%$ statistical power with a one-sided $\alpha$ level of 0.025 , a sample size of 150 evaluable patients per group was 
needed. Assuming a $10 \%$ drop-out rate, 165 patients per group needed to be enrolled. To assess non-inferiority for the primary endpoint of a CR rate at $120 \mathrm{~h}$, the lower boundary of the two-sided $95 \%$ confidence interval (CI) for the difference (1-day dexamethasone minus 3-day dexamethasone) between the $\mathrm{CR}$ rates in the two treatment arms was compared with the preset threshold.

The primary endpoint was analyzed for both the ITT and PP cohort, and the results were interpreted in a confirmatory manner to demonstrate the non-inferiority hypothesis. The PP cohort was used for all secondary efficacy analyses including the primary endpoint analyzed in the multivariable context. The data analyses were carried out by an independent statistician who was blinded to study treatments.

Comparisons of CR during the acute phase (0-24 h), and delayed phase $(24-120 \mathrm{~h})$ were preplanned secondary analyses for either cohort. Consistency of treatment effect (CR in the overall study period) across gender (female versus male) and type of chemotherapy (anthracycline plus cyclophosphamide (AC)-based versus non-AC-based) were assessed by evaluating the first-order interactions between treatment and either risk factor, included one by one into a generalized linear model implemented with binomial distribution and identity link function. This non-canonical link function was adopted to estimate risk differences (RD) instead of the usual odds ratios. RD allowed a straightforward comparison between the unadjusted (univariable analysis) and adjusted (multivariable analysis) two-sided 95\% CI of between-group difference in CR to anti-emetic treatment for testing the non-inferiority.

A chi-square test was used to analyze the following: $\mathrm{CC}$ rates; proportion of patients with no emetic episodes, no nausea, or no rescue medication; and proportion of patients with nausea (worst severity as assessed by the Likert scale). Treatment-related adverse events occurring in at least $2 \%$ of patients were summarized descriptively by treatment group.

The analyses of all secondary endpoints were evaluated in an explorative or descriptive manner, and therefore no adjustment for multiplicity was performed. All $P$ values were two-sided, and a $P$ value $<0.05$ was considered statistically significant. All statistical analyses were performed using SAS software (version 9.1; SAS Institute, Cary, NC).

\section{Results}

Patient characteristics

From October 13, 2006 to June 6, 2008, 334 patients were randomized to receive palonosetron in combination with dexamethasone as either a single dose or for 3 days. Two patients did not receive study treatment (Fig. 1); thus, a total of 332 patients were included in the ITT cohort for efficacy analyses. Five patients were lost to follow-up (two patients in the study arm and three in the reference arm) and an additional three had a major protocol deviation (one patient in the study arm and two in the reference arm). Therefore, a total of 324 patients were evaluable for efficacy analysis in the PP cohort.

Demographic and clinical characteristics for the ITT cohort were similar between treatment groups (Table 1). The majority of the study population ( 225 of 332 patients $(67.8 \%))$ was less than 65 years of age, and most patients (216 of 332 patients $(65.1 \%)$ ) were women. The majority of patients were diagnosed with either breast (143 of 332 patients $(43.1 \%))$ or colorectal (121 of 332 patients $(36.4 \%))$ cancers. Of the chemotherapeutic treatments received on day 1 in the efficacy analyses, most patients received either oxaliplatin-based regimens (119 of 332 patients $(35.8 \%))$ or AC-containing regimens $(35.2 \%$ of the study population).

\section{Efficacy}

The proportion of patients in either the ITT or PP cohort achieving CR during the overall study period $(0-120 \mathrm{~h})$ after MEC is presented in Table 2. Non-inferiority of the 1-day regimen was demonstrated, as the lower boundaries of the $95 \%$ CI of the difference with the 3-day regimen were greater than the preset threshold of $-15 \%$ difference.

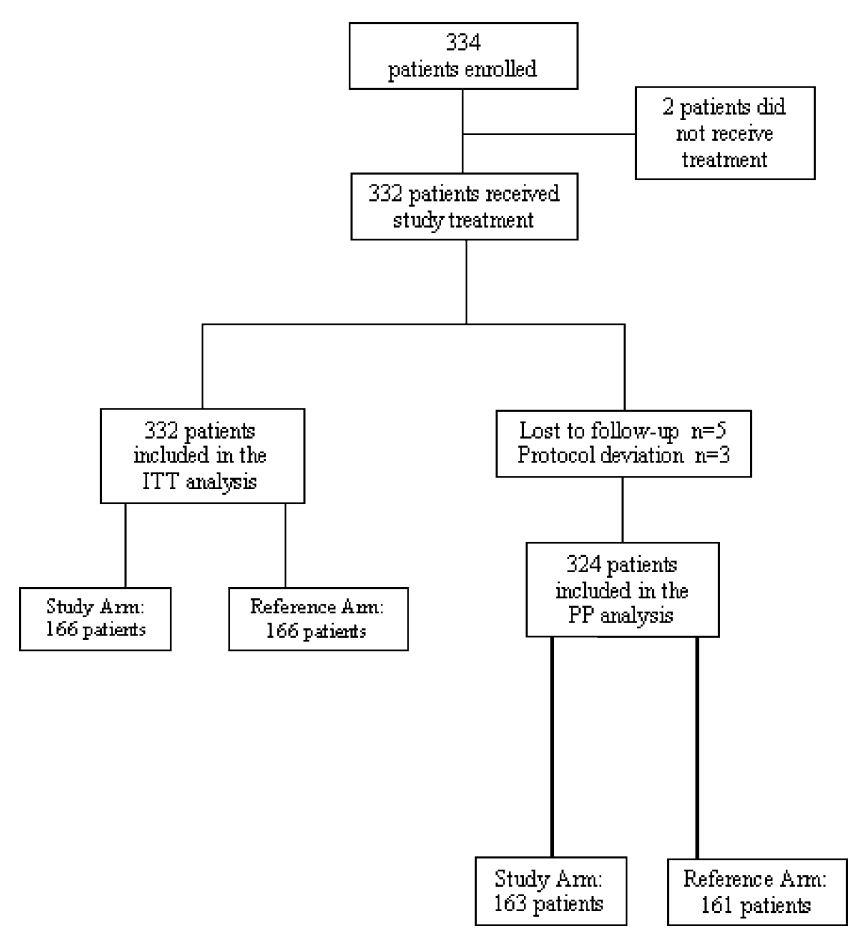

Fig. 1 Study flow chart. ITT intention-to-treat, $P P$ per-protocol 
Table 1 Baseline demographic and clinical characteristics in the intention-to-treat cohort
${ }^{a}$ Palonosetron plus dexamethasone

${ }^{\mathrm{b}}$ Eastern Cooperative Oncology Group

${ }^{c} 1-2$ glasses of wine

${ }^{\mathrm{d}}$ Anthracycline plus cyclophosphamide

\begin{tabular}{|c|c|c|}
\hline Variable & Palo plus 1-day $\operatorname{Dex}^{\mathrm{a}}(n=166)$ & Palo plus 3-day Dex $(n=166)$ \\
\hline \multicolumn{3}{|c|}{ Age categories $(n(\%))$} \\
\hline$<65$ years & $114(68.7)$ & $111(66.9)$ \\
\hline$\geq 65$ years & $52(31.3)$ & $55(33.1)$ \\
\hline Mean $( \pm \mathrm{SD})$ & $56.9( \pm 11.8)$ & $57.2( \pm 11.3)$ \\
\hline \multicolumn{3}{|l|}{ Body weight (kg) } \\
\hline Mean $( \pm \mathrm{SD})$ & $68.8( \pm 14.7)$ & $66.6( \pm 12.6)$ \\
\hline \multicolumn{3}{|l|}{ Height (cm) } \\
\hline Mean $( \pm \mathrm{SD})$ & $163.7( \pm 8.9)$ & $163.1( \pm 7.8)$ \\
\hline \multicolumn{3}{|l|}{ Gender $(n(\%))$} \\
\hline Female & $103(62)$ & $113(68.1)$ \\
\hline Male & $63(38)$ & $53(31.9)$ \\
\hline \multicolumn{3}{|l|}{ ECOG score $^{\mathrm{b}}(n(\%))$} \\
\hline 0 & $152(91.6)$ & $152(91.6)$ \\
\hline 1 & $14(8.4)$ & $13(7.8)$ \\
\hline 2 & 0 & $1(0.6)$ \\
\hline \multicolumn{3}{|l|}{ Tumor type $(n(\%))$} \\
\hline Breast & $65(39.2)$ & $78(47)$ \\
\hline Colorectal & $65(39.2)$ & $56(33.7)$ \\
\hline Lung & $15(9.0)$ & $13(7.8)$ \\
\hline Other & $21(12.6)$ & $19(11.5)$ \\
\hline \multicolumn{3}{|c|}{ Alcohol consumption $(n(\%))$} \\
\hline No & $101(60.8)$ & $99(59.6)$ \\
\hline Everyday $^{\mathrm{c}}$ & $65(39.2)$ & $67(40.4)$ \\
\hline \multicolumn{3}{|l|}{ Tumor stage $(n(\%))$} \\
\hline Early & $98(59)$ & $115(69.3)$ \\
\hline Metastatic & $68(41)$ & $51(30.7)$ \\
\hline \multicolumn{3}{|c|}{ Chemotherapy (n (\%)) } \\
\hline AC-based ${ }^{\mathrm{d}}$ & $54(32.5)$ & $63(38)$ \\
\hline Oxaliplatin-based & $64(38.6)$ & $55(33.1)$ \\
\hline Carboplatin-based & $21(12.7)$ & $16(9.6)$ \\
\hline Irinotecan-based & $14(8.4)$ & $15(9.1)$ \\
\hline Other & $13(7.8)$ & $17(10.2)$ \\
\hline
\end{tabular}

For the acute (0-24 h) and delayed (24-120 h) phases, palonosetron plus 1-day dexamethasone was non-inferior to the 3-day regimen. Secondary efficacy analyses are presented in Table 3. During the delayed phase, significantly fewer patients receiving the 1-day dexamethasone regimen required no rescue medication compared with those who received the 3-day regimen. When the proportion of patients with no emesis or nausea was assessed daily, there were also no statistically significant differences between groups at any time point after chemotherapy initiation (data not shown).

Subset analyses of each treatment group by gender and type of chemotherapy administered were done for CR during the acute and delayed phases (Table 4). Lower rates of CR were observed for palonosetron plus single-dose dexamethasone compared with palonosetron plus 3-day dexamethasone in women during the delayed phase (24-
$120 \mathrm{~h}) . \mathrm{CR}$ rates in the subgroup of patients receiving nonAC MEC regimens showed no difference between treatment groups in the acute and delayed phases. Fewer patients receiving $\mathrm{AC}$-containing regimens achieved $\mathrm{CR}$ in the 1-day regimen with respect to those in the 3-day regimen during the delayed phase. It should be noted that half of the female patients (1-day regimen, 49.5\%; 3-day regimen, 52\%) in either arm received AC-containing chemotherapy. There were no significant interactions between treatment and either gender $(P=0.512)$ or type of chemotherapy $(P=0.672)$ in the overall study period for the primary endpoint of $\mathrm{CR}$. On univariable analysis only female gender (RD 14.7\%; 95\% CI, 4.7 to $24.7 \%$; $P=$ 0.004 ) and AC-containing chemotherapy (RD 12.8\%; 95\% CI, 2.0 to $23.6 \% ; P=0.020)$ were associated with worse outcome in terms of overall $\mathrm{CR}$ to anti-emetic treatment. Nevertheless such associations were no longer statistically 
Table 2 Complete response (defined as no emetic episodes and no use of rescue medication) rates in the study cohorts

\begin{tabular}{|c|c|c|c|c|}
\hline & \multicolumn{3}{|l|}{ Complete responses } & \multirow[b]{2}{*}{$P^{\mathrm{c}}$} \\
\hline & Palo plus 1-day $\operatorname{Dex}^{\mathrm{a}}(n(\%))$ & Palo plus 3-day Dex (n (\%)) & $\begin{array}{l}\text { Difference between groups }{ }^{b} \\
(95 \% \mathrm{CI}), \%\end{array}$ & \\
\hline ITT cohort $^{\mathrm{d}}, n=332$ & $n=166$ & $n=166$ & & \\
\hline Acute phase $(0-24$ h) & $147(88.6)$ & $140(84.3)$ & $4.2(-3.1$ to 11.6$)$ & 0.262 \\
\hline $95 \% \mathrm{CI}$ & 82.7 to 92.9 & 77.9 to 89.5 & & \\
\hline Delayed phase $(24-120$ h) & $114(68.7)$ & $129(77.7)$ & $-9.0(-18.5$ to 0.4$)$ & 0.116 \\
\hline $95 \% \mathrm{CI}$ & 61.0 to 75.6 & 70.6 to 83.8 & & \\
\hline Overall phase $^{\mathrm{e}}(0-120 \mathrm{~h})$ & $112(67.5)$ & $118(71.1)$ & $-3.6(-13.5$ to 6.3$)$ & ND \\
\hline $95 \% \mathrm{CI}$ & 59.8 to 74.5 & 63.6 to 77.9 & & \\
\hline PP cohort $\mathrm{f}^{\mathrm{f}} n=324$ & $(n=163)$ & $(n=161)$ & & \\
\hline Acute phase $(0-24$ h) & $144(88.3)$ & $135(83.9)$ & $4.5(-3$ to 12$)$ & 0.242 \\
\hline $95 \% \mathrm{CI}$ & 82.4 to 92.8 & 77.2 to 89.2 & & \\
\hline Delayed phase $(24-120$ h) & $112(68.7)$ & $124(77.0)$ & $-8.3(-17.9$ to 1.3$)$ & 0.093 \\
\hline $95 \% \mathrm{CI}$ & 60.9 to 75.7 & 69.7 to 83.3 & & \\
\hline Overall phase $^{\mathrm{e}}(0-120$ h $)$ & $109(66.9)$ & $113(70.2)$ & $-3.3(-13.4$ to 6.8$)$ & ND \\
\hline $95 \% \mathrm{CI}$ & 59.1 to 74.0 & 62.5 to 77.1 & & \\
\hline \multicolumn{5}{|l|}{$N D$ not done } \\
\hline \multicolumn{5}{|l|}{${ }^{a}$ Palonosetron plus dexamethasone } \\
\hline \multicolumn{5}{|c|}{${ }^{\mathrm{b}} 1$-day minus 3-day regimen with $95 \%$ confidence interval obtained using normal approximation of binomial data } \\
\hline \multicolumn{5}{|c|}{${ }^{\mathrm{c}}$ Two-sided chi-square test (1-day vs. 3-day) } \\
\hline \multicolumn{5}{|c|}{${ }^{\mathrm{d}}$ Intention-to-treat } \\
\hline \multicolumn{5}{|c|}{$\begin{array}{l}\text { e Non-inferiority hypothesis in primary analysis was proven as the lower boundaries of the } 95 \% \mathrm{CI} \text { of between-group difference were greater than the preset } \\
\text { threshold }(-15 \%)\end{array}$} \\
\hline
\end{tabular}

significant in a multivariable analysis. On both univariable and multivariable analysis the lower boundaries of the $95 \%$ CI of between-group difference were greater than the preset threshold of $-15 \%$ (univariable: RD $-3.3 \% ; 95 \% \mathrm{CI},-13.4$ to $6.8 \% ; P=0.520$; multivariable: $\mathrm{RD}-4.4 \% ; 95 \% \mathrm{CI}$, -14.1 to $5.4 \% ; P=0.381)$. Therefore, the non-inferiority hypothesis of the 1-day regimen was proven also after adjusting for the model covariates.

The severity of nausea was not significantly different between groups for each study day or during the delayed and overall phases. However, this was only an exploratory analysis due to the unblinded design of the study. The majority of nausea was mild in severity during the delayed phase (1-day regimen, 33.7\%; 3-day regimen 29.2\%); very few patients reported nausea of at least moderate severity on any study day in the 1-day regimen $(2.4 \%$ to $4.9 \%$ of patients) or in the 3 -day regimen $(3.1 \%$ to $6.8 \%$ of patients). In both groups, very few patients experienced severe nausea within $24 \mathrm{~h}$ ( 0 of 163 patients in the 1-day regimen and four of 161 patients $(2.5 \%)$ in the 3 -day regimen). Only one patient receiving the 1-day regimen had severe nausea $120 \mathrm{~h}$ after chemotherapy initiation.
Tolerability

A total of 332 patients were evaluable for safety. Overall, 159 patients $(47.9 \%)$ reported one or more adverse events (both related and unrelated to study treatment), 82 patients $(49.4 \%)$ in the 1 -day regimen and 77 patients $(46.4 \%)$ in the 3-day regimen. There were no clinically relevant differences between groups with respect to the overall incidence of adverse events. The most common treatmentrelated adverse events in either treatment group were headache (1-day regimen, 19.9\%; 3-day regimen, 13.9\%) followed by constipation $(9.6 \%$ and $10.8 \%$, respectively) and fatigue $(9.0 \%$ and $4.2 \%$, respectively). No serious adverse events occurred during the study.

\section{Discussion}

In the current phase III trial, the primary hypothesis of noninferiority of palonosetron plus single-dose dexamethasone compared with 3-day dexamethasone regimen in the prevention of CINV following a broad range of MEC 
Table 3 Secondary efficacy analyses in the per-protocol cohort

\begin{tabular}{|c|c|c|c|}
\hline Variable & Palo plus 1-day $\operatorname{Dex}^{\mathrm{a}}(n=163), n(\%)$ & Palo plus 3-day Dex $(n=161), n(\%)$ & Difference $^{\mathrm{b}}, 95 \%$ CI (\%) \\
\hline \multicolumn{4}{|l|}{ Complete control $^{\mathrm{c}}$} \\
\hline Acute phase $(0-24$ h) & $142(87.1)$ & $132(82.0)$ & $5.1(-2.7$ to 13$)$ \\
\hline Delayed phase (24-120 h) & $108(66.3)$ & $122(75.8)$ & $-9.5(-19.3$ to 0.3$)$ \\
\hline Overall phase $(0-120 \mathrm{~h})$ & $105(64.4)$ & $109(67.7)$ & $-3.3(-13.6$ to 7$)$ \\
\hline \multicolumn{4}{|l|}{ No emetic episodes } \\
\hline Acute phase (0-24 h) & $153(93.9)$ & $148(91.9)$ & $1.9(-3.7$ to 7.5$)$ \\
\hline Delayed phase $(24-120 \mathrm{~h})$ & $140(85.9)$ & $145(90.1)$ & $-4.2(-11.2$ to 2.9$)$ \\
\hline Overall phase $(0-120 \mathrm{~h})$ & $134(82.2)$ & $135(83.9)$ & $-1.6(-9.8$ to 6.5$)$ \\
\hline \multicolumn{4}{|l|}{ No nausea } \\
\hline Acute phase $(0-24$ h) & $128(78.5)$ & $117(72.7)$ & $5.9(-3.5$ to 15.2$)$ \\
\hline Delayed phase $(24-120 \mathrm{~h})$ & $93(57.1)$ & $100(62.1)$ & $-5.1(-15.7$ to 5.6$)$ \\
\hline Overall phase $(0-120 \mathrm{~h})$ & $85(52.1)$ & $91(56.5)$ & $-4.4(-15.2$ to 6.5$)$ \\
\hline \multicolumn{4}{|l|}{ No use of rescue medication } \\
\hline Acute phase $(0-24$ h $)$ & $148(90.8)$ & $144(89.4)$ & $1.4(-5.1$ to 7.9$)$ \\
\hline Delayed phase (24-120 h) & $119(73.0)$ & $134(83.2)^{*}$ & $-10.2(-19.2$ to -1.3$)$ \\
\hline Overall phase $(0-120 \mathrm{~h})$ & $116(71.2)$ & $129(80.1)$ & $-9(-18.3$ to 0.3$)$ \\
\hline
\end{tabular}

* $P=0.026$ (two-sided Chi-square test (1-day vs. 3-day))

a palonosetron plus dexamethasone

${ }^{\mathrm{b}}$ 1-day minus 3-day regimen with $95 \%$ confidence interval obtained using normal approximation of binomial data

${ }^{\mathrm{c}}$ No emetic episodes, no use of rescue medication, and no more than mild nausea

regimens was shown for CR during the overall phase (0$120 \mathrm{~h}$ post-chemotherapy).

In patients receiving MEC, current guidelines recommend a combination of a 5- $\mathrm{HT}_{3}$-receptor antagonist and dexamethasone before chemotherapy initiation, with either agent given alone on days 2 and 3 [3]. However, the use of dexamethasone in clinical practice remains limited because of concerns about potential side effects and/or other factors [5, 8].

Table 4 Subgroup analysis by gender and type of chemotherapy of the complete response (defined as no emetic episodes and no rescue medication) rates in the per-protocol cohort

Complete responses

\begin{tabular}{lll}
\hline Palo plus 1-day $\operatorname{Dex}^{\mathrm{a}}(n(\%))$ & Palo plus 3-day Dex $(n(\%))$ & ${ }^{\mathrm{b}}$ Difference, $95 \%$ CI $(\%)$
\end{tabular}

\begin{tabular}{|c|c|c|c|}
\hline \multicolumn{4}{|c|}{ Acute phase $(0-24$ h) Gender } \\
\hline Women & 88/101 (87.1) & $85 / 109(78)$ & $9.1(-1$ to 19.3$)$ \\
\hline Men & $54 / 62(87.1)$ & 47/52 (90.4) & $-3.3(-14.9$ to 8.3$)$ \\
\hline \multicolumn{4}{|l|}{ Chemotherapy } \\
\hline AC-based ${ }^{c}$ & $44 / 52(84.6)$ & 45/61 (73.8) & $10.8(-3.9$ to 25.6$)$ \\
\hline Non-AC-based & 98/111 (88.3) & $87 / 100(87.0)$ & $1.3(-7.6$ to 10.2$)$ \\
\hline \multicolumn{4}{|c|}{ Delayed phase $(24-120$ h) Gender } \\
\hline Women & $62 / 101(61.4)$ & $81 / 109(74.3)^{*}$ & $-12.9(-25.5$ to -0.4$)$ \\
\hline Men & $46 / 62(74.2)$ & $41 / 52(78.8)$ & $-4.7(-20.2$ to 10.9$)$ \\
\hline \multicolumn{4}{|l|}{ Chemotherapy } \\
\hline AC-based ${ }^{\mathrm{c}}$ & $29 / 52(55.8)$ & $46 / 61(75.4)^{* *}$ & $-19.6(-36.9$ to -2.3$)$ \\
\hline Non-AC-based & 79/111 (71.2) & $76 / 100(76.0)$ & $-4.8(-16.7$ to 7$)$ \\
\hline
\end{tabular}

$* P=0.045$ (two-sided chi-square test (1-day vs. 3 -day)); $* * P=0.028$

${ }^{a}$ Palonosetron plus dexamethasone

${ }^{\mathrm{b}}$ 1-day minus 3-day regimen with $95 \%$ confidence interval obtained using normal approximation of binomial data

${ }^{\mathrm{c}}$ Anthracycline plus cyclophosphamide 
The second-generation 5- $\mathrm{HT}_{3}$-receptor antagonist palonosetron has been recently reported to lead to receptor alteration or internalization resulting in a extended inhibition of receptor function [16]. Two phase III trials have demonstrated that palonosetron alone is effective in preventing both acute and delayed CINV following MEC $[11,12]$.

Following the design and methodology of the pivotal phase III trials, we have explored the hypothesis that additional dexamethasone doses beyond day 1 of MEC administration may not be necessary in patients receiving palonosetron. In the current study, which included a heterogeneous patient population receiving commonly administered MEC regimens to reflect actual clinical practice, CR rates were $89 \%$ in the 1-day regimen arm versus $84 \%$ in the 3 -day regimen arm during the acute phase. Response rates were $68 \%$ versus $71 \%$, respectively, for complete protection against CINV during the overall study period. However, analysis of the components of CR, vomiting and use of rescue medication, showed that fewer patients taking a single dexamethasone dose reported no rescue medication use during the delayed phase. The added benefit for this secondary endpoint in patients taking additional dexamethasone doses is most likely related to the characteristics of the study population. Women accounted for the majority of patients in each treatment group, and half of the female patients in either arm received AC-containing chemotherapy. This may bias the antiemetic protection against delayed CINV in the patient cohort receiving no additional dexamethasone less favorably than cohort taking dexamethasone on days 2 and 3. A subgroup analysis showed that the standard 3-day regimen was better than the 1-day regimen for complete protection against delayed CINV in women $(61.4 \%$ versus $74.3 \%$; $P=$ $0.045)$. It is well known that women who receive ACcontaining chemotherapy have a particularly high risk of developing CINV, but at the time the current trial was planned, the AC-based regimens were considered as MEC [17]. Within the AC subgroup, the standard regimen appeared to be more efficacious in the delayed phase in comparison with the 1-day regimen $(\mathrm{CR}, 55.8 \%$ versus $75.4 \% ; P=0.028$ ). A recently reported double-blind, phase III trial evaluated the efficacy of palonosetron plus 1versus 3-day dexamethasone in chemotherapy-naïve female breast cancer patients $(n=300)$ receiving anthracycline and/ or cyclophosphamide-containing chemotherapy [18]. Noninferiority between the two treatments was demonstrated by similar CR rates in the overall period (study primary endpoint) but, interestingly, patients receiving the 3-day dexamethasone regimen experienced on day 3 less emesis than the group receiving a single dose of dexamethasone ( $89 \%$ versus $97 \%, P=0.004$ ). In the current study, on univariable analysis female gender and AC-containing chemotherapy were significantly associated with worse outcome. Such associations were no longer statistically significant in a multivariable analysis, but the trial was not powered to enable careful assessment of whether there is any difference in efficacy from gender and the type of chemotherapy. In patients with breast cancer receiving ACcontaining chemotherapy, the MASCC/ESMO anti-emetic guidelines, recently updated in 2010 , recommend a $5-\mathrm{HT}_{3}$ receptor antagonist in combination with dexamethasone and an neurokinin-1 receptor antagonist (aprepitant or fosaprepitant) to prevent acute CINV, with aprepitant given alone on days 2 and 3 for prevention of delayed symptoms [19]. In a phase II trial evaluating the efficacy of palonosetron plus 3-day dexamethasone and aprepitant in 58 patients receiving MEC (including AC-containing chemotherapy in $41 \%$ of cases), $78 \%$ of patients achieved CR during the delayed and overall phases [20]. More recently, results from a phase II study that included 41 patients (40 were female) receiving MEC (including AC-containing chemotherapy in $90 \%$ of cases) indicated that a single-day regimen of palonosetron, dexamethasone and aprepitant is feasible and effective for protection against acute and delayed vomiting [21]. In the light of this, a single-day three-drug anti-emetic regimen should be formally compared with the standard multi-day regimen in female patients for the prevention of CINV following AC-containing chemotherapy.

We acknowledge that as the present trial was unblinded this could have potentially influenced the results. However, the consistency of the efficacy results observed in the 1-day regimen arm with those previously reported on palonosetron supports the validity of the current study [13, 15, 22].

In conclusion, we provide evidence that the combination of palonosetron and dexamethasone given on day 1 of common MEC regimens is non-inferior to the same regimen with dexamethasone also administered on days 2 and 3 for the complete protection against acute and delayed CINV. It is important to remember that the current study was not designed to address whether the 1-day regimen should be pursued or not according to the type of MEC chemotherapy. An unplanned subgroup analysis suggested that the major benefit of the 1-day regimen occurs in patients receiving non-AC-containing chemotherapy $(65 \%$ of the study population). Therefore, in patients receiving MEC other than AC-based regimens, this dexamethasone sparing regimen minimizes medication administration that patients must remember at home and provides the potential to improve both adherence with anti-emetic prophylaxis and quality of life.

Acknowledgements The authors thank the following clinicians who participated in the study:

Federica Villa, Ospedale San Gerardo, Monza, Italy; Maria Teresa Cattaneo, Ospedale L. Sacco, Milan, Italy; Michelangelo Russillo, Istituto Regina Elena, Rome, Italy; Raffaele Venezia, Ospedale 
Serbelloni, Gorgonzola, Italy; Claudia Biasini, Ospedale Guglielmo da Saliceto, Piacenza, Italy; Fabio Malugani, Ospedale di Sondrio, Sondrio, Italy; Rodolfo Passalacqua, Silvia Lazzarelli, Istituti Ospitalieri, Cremona, Italy; Luigi Dogliotti, Lucianna Russo, Ospedale San Luigi Gonzaga, Orbassano, Italy; Alessandra Galetto, David Rondonotti, Ospedale Maggiore della Carità, Novara, Italy; Enrico Aitini, Maria Antonietta Perrone, Ospedale C. Poma, Mantova, Italy; Graziella Pinotti, Giovanni Giardina, Ospedale di Circolo, Varese, Italy; Ignazio Carreca, Dario Piazza, Policlinico P. Giaccone, Palermo, Italy.

The authors thank the patients, nurses, and data managers who participated in this trial. We also thank Italfarmaco S.p.A., Milan, Italy for the generous gift of palonosetron.

Presented in part at the 10th Annual Meeting of the Italian Association of Medical Oncology, Verona, Italy, October 2008.

Conflict of interest The study was not funded. There were no financial disclosures from any authors.

Open Access This article is distributed under the terms of the Creative Commons Attribution Noncommercial License which permits any noncommercial use, distribution, and reproduction in any medium, provided the original author(s) and source are credited.

\section{References}

1. Sharma R, Tobin P, Clarke SJ (2005) Management of chemotherapy-induced nausea, vomiting, oral mucositis, diarrhoea. Lancet Oncol 6:93-102. doi:10.1016/S1470-2045(05)01735-3

2. Grunberg SM (2007) Antiemetic activity of corticosteroids in patients receiving cancer chemotherapy: dosing, efficacy, and tolerability analysis. Ann Oncol 18:233-240

3. Hesketh PJ (2008) Chemotherapy-induced nausea and vomiting. N Engl J Med 358:2482-2494

4. Hesketh PJ, Kris MG, Grunberg SM et al (1997) Proposal for classifying the acute emetogenicity of cancer chemotherapy. J Clin Oncol 15:103-109

5. Roila F, Hesketh PJ, Herrstedt J et al (2006) Prevention of chemotherapy- and radiotherapy-induced emesis: results of the 2004 Perugia International Antiemetic Consensus Conference. Ann Oncol 17:20-28. doi:10.1093/annonc/mdj078

6. Naeim A, Dy SM, Lorenz KA, Sanati H, Walling A, Asch SM (2008) Evidence-based recommendations for cancer nausea and vomiting. J Clin Oncol 26:3903-3910. doi:10.1200/JCO.2007.15.9533

7. Ioannidis JP, Hesketh PJ, Lau J (2000) Contribution of dexamethasone to control of chemotherapy-induced nausea and vomiting: a meta-analysis of randomized evidence. J Clin Oncol 18:3409-3422

8. Vardy J, Chiew KS, Galica J, Pond GR, Tannock IF (2006) Side effects associated with the use of dexamethasone for prophylaxis of delayed emesis after moderately emetogenic chemotherapy. $\mathrm{Br}$ J Cancer 94:1011-1015. doi:10.1038/sj.bjc.6603048

9. Geling O, Eichler H-G (2005) Should 5-hydroxytryptamine-3 receptor antagonists be administered beyond 24 hours after chemotherapy to prevent delayed emesis? Systematic reevaluation of clinical evidence and drug cost implications. J Clin Oncol 23:1289-1294. doi:10.1200/JCO.2005.04.022
10. Siddiqui MA, Scott LJ (2004) Palonosetron. Drugs 64:1125-1132

11. Gralla R, Lichinitser M, Van Der Vegt S et al (2003) Palonosetron improves prevention of chemotherapy-induced nausea and vomiting following moderately emetogenic chemotherapy: results of a double-blind randomized phase III trial comparing single doses of palonosetron with ondansetron. Ann Oncol 14:1570-1577. doi:10.1093/annonc/mdg417

12. Eisenberg P, Figueroa-Vadillo J, Zamora R et al (2003) Improved prevention of moderately emetogenic chemotherapy-induced nausea and vomiting with palonosetron, a pharmacologically novel 5-HT3 receptor antagonist: results of a phase III, singledose trial versus dolasetron. Cancer 98:2473-2482. doi:10.1002/ cncr. 1187

13. Rubenstein EB, Gralla RJ, Eisenberg P, et al (2003) Palonosetron (PALO) compared with ondansetron (OND) or dolasetron (DOL) for prevention of acute and delayed chemotherapy-induced nausea and vomiting (CINV). Combined results of two Phase III trials. Proc Am Soc Clin Oncol 22:729. Available at: http://www.asco. org/ASCO/Abstracts. Accessed 19 February 2009

14. Roila F, Warr D, Clark-Snow RA et al (2005) Delayed emesis: moderately emetogenic chemotherapy. Support Care Cancer 13:104-108. doi:10.1007/s00520-004-0700-8

15. Hajdenberg J, Grote T, Yee L, Arevalo-Araujo R, Latimer LA (2006) Infusion of palonosetron and dexamethasone for the prevention of chemotherapy-induced nausea and vomiting. J Support Oncol 4:467-471

16. Rojas C, Stathis M, Thomas AG et al (2008) Palonosetron exhibits unique molecular interactions with the 5-HT3 receptor. Anesth Analg 107:469-478. doi:10.1213/ane.06013e318172fa74

17. Warr DG, Hesketh PJ, Gralla RJ et al (2005) Efficacy and tolerability of aprepitant for the prevention of chemotherapyinduced nausea and vomiting in patients with breast cancer after moderately emetogenic chemotherapy. J Clin Oncol 23:28222830. doi: $10.1200 / J C O .2005 .09 .050$

18. Aapro M, Fabi A, Nolè F et al (2010) Double-blind, randomised, controlled study of the efficacy and tolerability of palonosetron plus dexamethasone for 1 day with or without dexamethasone on days 2 and 3 in the prevention of nausea and vomiting induced by moderately emetogenic chemotherapy. Ann Oncol 21:1083-1088. doi:10.1093/annonc/mdp584

19. MASCC/ESMO Antiemetic Guidelines. Available at: http://data. memberclicks.com/site/mascc/MASCC_Guidelines_English_ 2010.pdf. Accessed 31 May 2010

20. Grote T, Hajdenberg J, Cartmell A, Ferguson S, Ginkel A, Charu V (2006) Combination therapy for chemotherapy-induced nausea and vomiting in patients receiving moderately emetogenic chemotherapy: palonosetron, dexamethasone, and aprepitant. J Support Oncol 4:403-408

21. Grunberg SM, Dugan M, Muss H et al (2009) Effectiveness of a single-day three-drug regimen of dexamethasone, palonosetron, and aprepitant for the prevention of acute and delayed nausea and vomiting caused by moderately emetogenic chemotherapy. Support Care Cancer 17:589-594. doi:10.1007/s00520-008-0535-9

22. Giuliani F, Cilenti G, Nugnes I et al (2008) Palonosetron for prevention of acute and delayed nausea and vomiting induced by moderately emetogenic adjuvant folfox-4 regimen in colorectal cancer (CRC) patients: a phase II study of the Gruppo Oncologico dell'Italia Meridionale (GOIM). Eur J Cancer Suppl 6:102-106. doi:10.1016/j.ejcsup.2008.06.018 